\title{
Approach to the Seamless Management of Inflammatory Bowel Disease, Considering Special Situations, Shared Decision-Making, and Disease Burden
}

\author{
Nobuo Aoyama ${ }^{a}$ Toshiaki Shimizu ${ }^{b}$ \\ ${ }^{a}$ Aoyama Medical Clinic, Kobe, Japan; ${ }^{b}$ Department of Pediatrics and Adolescent Medicine, Juntendo University \\ Graduate School of Medicine, Tokyo, Japan
}

\section{Keywords}

Inflammatory bowel disease $\cdot$ Special situations $\cdot$ Shared

decision-making $\cdot$ Disease burden $\cdot$ IBD-Disk

\begin{abstract}
Background: While the number of inflammatory bowel disease (IBD) patients has been steadily increasing, a lot of effective treatments have become available, including biological drugs. However, there still exists no effective treatment universally for all types of IBD patients. We have continuously discussed "Total Care" for IBD patients in the 3 consecutive annual meetings of the Japanese Gastroenterological Association from the 14th meeting to the 16th meeting in 2018-2020. Summary: In the 14th meeting, we summarized cross-sectional issues under the title "From Total Care to Microbiota" as for their subthemes. In the 15th meeting, under the title of "Consensus and Pitfalls in Special Situations," we discussed the thiopurine treatment for the patients in their childhood or pregnancy and lactation period as well as the cytapheresis treatment for elderly patients. In the 16th meeting, under the title "Future Issues in Special Situations: Including Disease Burden," we discussed about the issues in childhood-onset IBD, including transitional care
\end{abstract}

and the new option of combination of treatments in their adulthood and their disease burdens. Key Messages: "Total Care" for IBD patients should be considered from a broad perspective with shared decision-making. It is imperative to involve medical staff members for careful handling of a wide range of disease burdens.

(c) 2020 The Author(s)

Published by S. Karger AG, Basel

\section{Introduction}

The Japanese Gastroenterological Association dealt with 4 themes under the project titled "Core Symposiums" where main theme was continued to be discussed over 3 years. For Core Symposium 2: Inflammatory Bowel Disease (IBD), we have continuously discussed the main theme titled "Approach to the Seamless Management of Inflammatory Bowel Disease" in the 3 consecutive annual meetings from the 14th meeting in 2018 to the 16th meeting in 2020 (Appendix 1).

In the 14th meeting (2018, Tokyo), as the first year of the core symposium, we summarized cross-sectional issues under the title "From Total Care to Microbiota" as for their subthemes. In the 15th meeting (2019, Saga), un- 
Fig. 1. IBD treatment "Step classification" (2020): step-up treatment (from Step 1 to Steps 2-3) is considered for UC; on the other hand, early biologic treatment is associated with improved clinical outcomes in both adult and pediatric CD patients (accelerated step-up or top-down from Step 3). As many IBD patients were able to achieve mucosal healing by the treatment of Step 3, the proportion of Step 3 is increasing year by year. Many options should be considered for the maintenance therapy, not just continuation of induction therapy (positive switch, non-medical switch). IBD, inflammatory bowel disease; UC, ulcerative colitis; $\mathrm{CD}$, Crohn's disease.

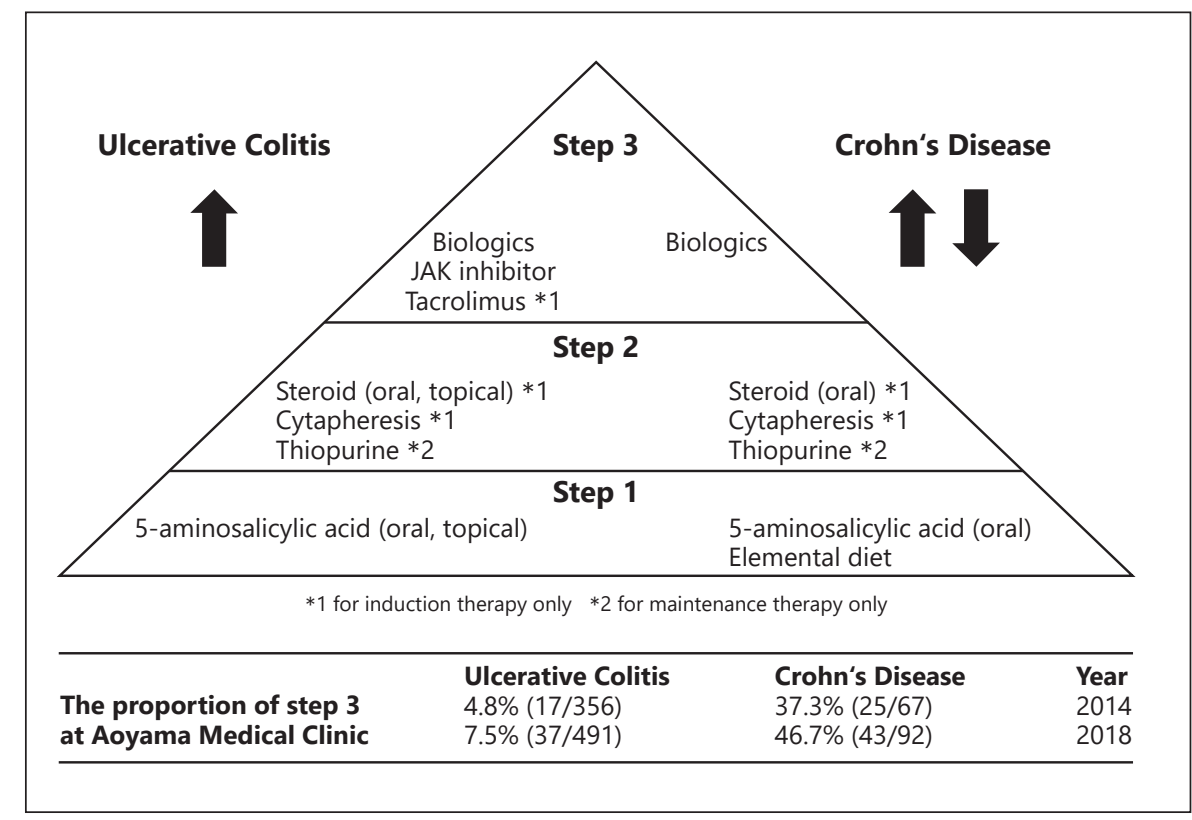

der the title "Consensus and Pitfalls in Special Situations," we mainly discussed the thiopurine treatment for the patients in their childhood or pregnancy and lactation period as well as the cytapheresis treatment for elderly patients. In the 16th meeting (2020, Himeji) as the final year, we mostly discussed about the issues in childhood-onset inflammatory bowel disease (IBD), including transitional care and the new option of combination of treatments in their adulthood and their disease burdens under the title "Future Issues in Special Situations: Including Disease Burden.”

\section{Special Situations}

The issues observed during patients' childhood, pregnancy, and elderly period have been gradually summarized (Appendix 2). There had been the recognition of the clinical characterizations of very early onset IBD. Recently, prospective survey evaluated the data of very early onset IBD patients diagnosed before 6 years of age from the time of diagnosis to 68 months after registration [1]. Genetic analysis was performed in $45 \%(72 / 160)$ of patients, and $30.6 \%(22 / 72)$ patients were diagnosed with immunodeficiency-associated IBD.

In 2017, the Japanese Society for Pediatric Gastroenterology, Hepatology and Nutrition published "Guidelines for autonomy support of patients with IBD in the transitional period" [2]. In its 16th meeting, the results of a questionnaire survey with IBD medical specialists regarding the transitional care from childhood to adulthood were reported. To be specific, $94 \%$ of the doctors who specialize in treating adults with digestive organ disease answered that patients were desired to be completely transferred to another department at a certain point, while $55 \%$ of the doctors who specialize in treating children with digestive organ disease answered that they were supposed to transfer to another department step-by-step with setting an overlapped period. In addition, concerning the age at which the patients are expected to be transferred to another department, $65 \%$ of the former doctors replied that it should be 16 , only $10 \%$ of the latter ones agreed with it, and $41 \%$ of them replied that it should be 18. These differences in their positions suggest that with an aim to pursue ideal transitions, it is important going forward to make discussions involving not only medical staff members further but also many other professionals from the fields of education, public administration, and so forth.

The risk of hepatosplenic T-cell lymphomas in young males (under the age of 35 years) being co-treated with thiopurines and anti-tumor necrosis factor agents can be reduced by limiting the duration of the combined treatment to 2 years (ECCO Statement 5E, 2015) [3]. Although thiopurine therapy may not be a risk factor for hematologic malignancies in Japanese adult patients with IBD [4], further increases in the number of cases in infant or childhood are necessary to conclude this important issue. 
Fig. 2. Shared decision-making and disease burden. Even in remission when physical QOL decline is mild, IBD patients strongly feel a mental burden; thus, they should be given the appropriate care including QOL and mental issues. It is imperative to involve medical staff members for shared decision-making and careful handling of a wide range of disease burdens. QOL, quality of life; IBD, inflammatory bowel disease.
Options of treatment

Impaired QOL related to physical symptoms

Defecation, Abdominal pain, etc.

Metal issues

Long term prognosis, drug safety, etc.

Shared decision making

Special situations

Useful tool such as IBD-DISK

Disease burden

Loss of health due to diseases

Broad perspective

Involve medical staff members for carefully handling

The positive risk of the thiopurine use for pregnant females (Category D), according to Food and Drug Administration (FDA), is considered to be low, and most drugs (including thiopurine) used for the treatment of IBD are considered to be of low risk during pregnancy, except for methotrexate and thalidomide (ECCO Statement 5B, 2015) [5]. Treatment guidelines considering pregnancy and childbirth in female patients with systemic lupus erythematosus, rheumatoid arthritis, juvenile idiopathic arthritis, and IBD were published in Japan in 2018, and they showed the thiopurine use for the pregnant IBD female are considered to be of low risk. The insurance coverage for thiopurine, tacrolimus, and cyclosporine use in pregnant females has been changed from contraindication to being used only when its benefits exceed its risks in Japan since 2018.

In the 15th meeting, we comprehensively debated about the pros and cons of the administration of thiopurine to young male patients and pregnant female ones. Then, for both the groups of patients, 7 out of 10 symposists expressed the opinion that they would preferentially take another option over thiopurine, if any.

It has been pointed out that for elderly patients, attentions should be paid to complicating diseases including infectious ones although there is no difference in terms of the effects between the therapeutic effects of drugs and drug interactions and equivalents attributable to the existence of other diseases (ECCO 2017) [6] as reported in Japan $[7,8]$. In Japan, several reports suggested the safety of cytapheresis for elderly patients $[9,10]$, which was also presented in the 15th meeting, should be considered under the risk of COVID-19.

\section{New Options of Combination of Treatments}

Regarding the treatments for the patients with IBD, 5-aminosalicylic acid, steroid, tacrolimus, and cytapheresis are indicated only for the remission-induction therapy; thiopurine is indicated only for the remission-maintenance; and biologics and Janus kinase inhibitors are indicated for both the remission induction therapy and the remission maintenance therapy. Stepup treatment (from Step 1 to Steps 2-3) is considered for ulcerative colitis (UC); on the other hand, early biologic treatment is associated with improved clinical outcomes in both adult and pediatric Crohn's disease (CD) patients (accelerated step-up or top-down from Step 3) [11].

As many IBD patients were able to achieve mucosal healing by the treatment of Step 3, the proportion of Step 3 is increasing year by year (UC $4.8 \%$ [17/356] and CD $37.3 \%$ [25/67] in 2014, and UC 7.5\% [37/491] and CD 46.7\% [43/92] in 2018 at the Aoyama Medical Clinic). Many options should be considered for the maintenance therapy, not just continuation of induction therapy, although the initial drug has been found to be effective (positive switch, non-medical switch).

In the 16th meeting, effect of vedolizumab was reported as the remission maintenance therapy after the remission induction therapy for UC using tacrolimus. Additionally, for $\mathrm{CD}$ after the remission induction therapy using infliximab, the drug was switched to ustekinumab appropriately for the remission maintenance therapy although the initial treatment was found to be effective.

The factors to be considered in choosing a drug during the remission maintenance therapy are not only special 
situations and the cancer risk because of the long-term use of thiopurine and tumor necrosis factor inhibitors $[12,13]$, but also social ones such as administration methods, the number of hospital visits, and costs, leading to further discussions over a variety of treatment combinations. Considering the appearance of many biologics and Janus kinase inhibitors, lots of ongoing clinical trials, and further increasing number of therapy options, it is critical to find the best ones among many of these therapies according to the need of each patient with comprehensive care (Fig. 1).

\section{Shared Decision-Making and Disease Burden}

While the number of IBD patients has been steadily increasing, a lot of effective treatments have become available including biological drugs, enabling patients to receive high-quality therapies. However, there still exists no effective treatment universally for all types of IBD patients, and accordingly, individual IBD patients cannot always choose an appropriate treatment for them from the beginning (uncertainty of treatment). In addition, their order of priority varies in terms of the choice according to their life stages such as the childhood, pregnancy, and elderly period (special situations); the number of hospital visits; the visit duration at a medical institution; costs; and administration methods (diversity of value). Regarding the choice of a treatment, the shared decision-making becomes important.

Some studies suggest that IBD causes decrease in quality of life (QOL) [14, 15], and patients with IBD need to be given mental care [16]. Advancement in treatment enables us to provide high QOL and makes us think about mental care. In the 16th meeting, we had a report discussing the mental health care for the patients in their unstable adolescent period and the relationship between QOL and depression in patients with IBD [17]. Even in UC remission and when physical QOL decline is mild, women strongly feel a mental burden; thus, they should be given the appropriate care, and disease burden including QOL and mental care is an important point that medical staff and physicians should deal with together.

Burden of disease (disease burden) is a concept that was developed in the 1990s by the Harvard School of Public Health, and the World Bank and the World Health Organization (WHO) described it as "loss of health due to diseases (2004)." While it is effective to use the tool such as IBD Disk [18] for identifying disease burdens from such a broad perspective, it is imperative to involve medical staff members for careful handling of a wide range of disease burdens (Fig. 2).

\section{Conclusions}

"Total Care" for IBD patients should be considered from a broad perspective with shared decision-making. It is imperative to involve medical staff members for careful handling of a wide range of disease burdens.

\section{Acknowledgements}

We are grateful to Prof. Hiroyuki Kato, Prof. Kazuma Fujimoto, and Prof. Yoshikazu Kinoshita, who kindly gave us the opportunity to chair Core Symposium 2 at the 14th, 15th, and 16th meetings.

\section{Statement of Ethics}

This study does not require ethical review.

\section{Conflict of Interest Statement}

The authors declare no conflicts of interest regarding this review article.

\section{Funding Sources}

This study has not been funded.

\section{Author Contributions}

T.S. and N.A.: conception and design and drafting and revision of the manuscript. All authors critically appraised the final manuscript. All authors gave final approval of the version to be published and agreed to be accountable for all aspects of the work in ensuring that questions related to the accuracy or integrity of any part of the work are appropriately investigated and resolved. 


\section{Appendix 1. Core Symposium 2: Inflammatory Bowel Disease (IBD): 2018-2020}

The 14th Annual Meeting of the Japanese Gastroenterological Association

Subtheme: From Total Care to Microbiota

Chairpersons: T. Shimizu (Juntendo University) and N. Aoyama (Aoyama Medical Clinic)

Time: 13:20-15:20, Friday, February 9, 2018 (Tokyo)

Eleven oral presentations

1. Holistic approach in surgical management for pediatric inflammatory bowel disease (IBD) (Uchida et al., Mie University).

2. Long-term effect of infliximab in children with ulcerative colitis (Shimizu et al., National Center for Child Health and Development).

3. A study of the colonoscopy system required for infant IBD (Furukawa et al., Niigata City General Hospital).

4. About the influence of the activity of pregnancy complicated by ulcerative colitis on pregnancy and childbirth (Ito et al., Tokyo Women's Medical University).

5. Prospective study of intestinal and oral cavity flora and prognosis of ulcerative colitis during clinical remission (Ohno et al., Keio University).

6. Characteristics of mucosa-associated microbiota in IBD (Nishino et al., Shiga University).

7. Combination with fecal microbial transplantation and antibiotics for ulcerative colitis (Ishikawa et al., Juntendo University).

8. IBD practice using shared decision-making (Yoshida et al., Ofuna Chuo Hospital).

9. Nurses' participation in the treatment decision-making process of IBD (Nunotani et al., Mukogawa Women's University).

10. The present conditions of regional alliances in ulcerative colitis. From surgical viewpoint (Ikeuchi et al., Hyogo College of Medicine).

11. The role of IBD specialty practitioner (IBD-p) in Total Care of patients with IBD and efforts of IBD medical cooperation in Kyoto and Shiga (Obata et al., Obata Medical Clinic).

The 15th Annual Meeting of the Japanese Gastroenterological Association

Subtheme: Consensus and Pitfalls in Special Situations

Chairpersons: N. Aoyama (Aoyama Medical Clinic) and T. Osada (Juntendo University)

Time: 14:20-17:00, Saturday, February 2, 2019 (Saga)

One keynote lecture and 10 oral presentations and general discussion

Keynote lecture

Use of thiopurine in childhood and adolescent IBD patients (Shimizu et al., National Center for Child Health and Development)

Oral presentations

1. Anti-TNF monotherapy for Crohn's disease anal fistula of younger male patients (Masuda et al., Ikoma Gastrointestinal Proctology Clinic).

2. Safety of thiopurine in pregnant women with IBD (Ikeda et al., Yokohama City University Medical Center).

3. Safety of biologics in pregnant women with IBD (Ooi et al., Kobe University).
4. The clinical usefulness of 6-TGN monitoring for ulcerative colitis using AZA/6-MP (Yamamoto et al., Kariya Toyota General Hospital).

5. Efficacy of cytapheresis in ulcerative colitis patients in special situations (Shibuya et al., Juntendo University).

6. A study of long-term prognosis after leukocyte removal therapy for ulcerative colitis (Yamasaki et al., Fujioka Hospital).

7. Optimization and team approach of cytapheresis in elderly patients with ulcerative colitis (Yokoyama et al., Hyogo College of Medicine).

8. Clinical characteristics and adequateness of single-needle GMA in moderate-severe active ulcerative colitis (Fukuchi et al., Iseikai Hospital).

9. Efficacy and safety of granulocyte adsorption apheresis in elderly patients with ulcerative colitis (Ito et al., Tokyo Women's Medical University).

10. Renal dysfunction by tacrolimus in elderly ulcerative colitis (Kakimoto et al., Osaka Medical College).

The 16th Annual Meeting of the Japanese Gastroenterological Association

Subtheme: Future Issues in Special Situations: Including

Disease Burden

Chairpersons: T. Shimizu (Juntendo University) and N. Aoyama (Aoyama Medical Clinic)

Time: 13:30-15:20, Friday, February 8, 2020 (Himeji)

Twelve oral presentations

1. Risk factors and management of growth failure in children with Crohn's disease: a single center experience (Usami et al., National Center for Child Health and Development).

2. The effectiveness of MR enterography with diffusion-weighted imaging and apparent diffusion coefficient map for detecting and assessing IBD in children and adolescents (Oka et al., National Center for Child Health and Development).

3. The problem in surgical therapy for pediatric IBD (Uchida et al., Mie University).

4. Transitional care for patients with childhood-onset IBD $(\mathrm{Ku}-$ magai et al., Jichi Medical University).

5. Eight cases of early onset IBD patients seamlessly managed by the cooperation between pediatric surgery, pediatrics, and gastroenterology (Tsuzuki et al., Saitama Medical University).

6. Investigating pathogenesis of IBD and its novel treatment using genetic analysis for very early onset IBD (Ishige et al., Gunma University).

7. Maintenance therapy of vedolizumab after induction of remission by tacrolimus in patients with refractory ulcerative colitis (Ito et al., Tokyo Women's Medical University).

8. The current status of the immunosuppressive treatment including biologics for the IBD (ulcerative colitis, Crohn's disease, and intestinal Behcet's disease) in elderly people in our hospital (Suzuki et al., Nagoya City University).

9. Switching from anti-TNFa agents to ustekinumab in patients with perianal Crohn's disease (Ota Y, Ieda Hospital).

10. Pregnancy outcomes in patients with IBD (Yokoyama et al., Hyogo College of Medicine).

11. Impaired quality of life (QOL) and disturbed mental health in adolescents with IBD (Hirano et al., National Center for Child Health and Development).

12. Disease burden of patients with IBD: evaluation of QOL and depression (Takahashi et al., Aoyama Medical Clinic and Mukogawa Women's University).
16

Digestion 2021;102:12-17

DOI: $10.1159 / 000511481$
Aoyama/Shimizu 


\section{Appendix 2. Special Situations}

Infant and Childhood (IBD).

Recognition of very early onset inflammatory bowel disease

The risk of hepatosplenic T-cell lymphomas in young males exposed to thiopurines co-treated with anti-TNF agents (ECCO Statement 5E, 2015).

Guidelines for autonomy support of patients with IBD in the transitional period (Japanese Society for Pediatric Gastroenterology, Hepatology and Nutrition 2017).

Pregnancy

Positive risk of the thiopurine use in pregnant females (Category $\mathrm{D}$ according to Food and Drug Administration) is considered to be low (ECCO Statement 5B, 2015).

Infliximab and adalimumab cross the placenta, and it is better to stop these around gestational weeks 24-26 when considered appropriate (ECCO Statement 5D, 2015).
Treatment guidelines considering pregnancy and childbirth of female patients with systemic lupus erythematosus, rheumatoid arthritis, juvenile idiopathic arthritis, and IBD in Japan (2018).

The insurance coverage for thiopurine, tacrolimus, and cyclosporine use in pregnant females has been changed from contraindication to being used only when its benefits exceed its risks in Japan (2018).

\section{Elderly}

No evidence that the efficacy of medical treatment in elderly IBD patients differs from that in younger adult patients (ECCO Current Practice Position 8, 2017).

Infections and related serious complications are more common in the elderly IBD patients, emphasizing the need to follow immunization guidelines thoroughly (ECCO Current Practice Position $6,2017)$

Treatment guidelines for elderly ulcerative colitis (Ministry of Health, Labor and Welfare) in Japan (2018).

\section{References}

1 Kudo T, Fujii T, Maisawa S-I, Sasaki M, Uchida $\mathrm{K}$, Ida S, et al. A multicenter prospective survey on early-onset inflammatory bowel disease in Japan. Digestion. 2020;18:1-9.

2 Kumagai $\mathrm{H}$, Akiyama T, Abukawa D, Ida S, Inui A, Kudo T, et al. Guidelines for autonomy support of patients with IBD in the transitional period. Jpn J Pediatr Gastroenterol Hepatol Nutr. 2018;32:15-27.

3 Annese V, Beaugerie L, Egan L, Biancone L, Bolling C, Brandts C, et al. European evidence-based consensus: inflammatory bowel disease and malignancies. J Crohns Colitis. 2015;9(11):945-65.

4 Fukata N, Okazaki K, Omiya M, Matsushita $\mathrm{M}$, Watanabe M; The Members of the Ministry of Health and Welfare of Japan's Inflammatory Bowel Diseases Study Group. Hematologic malignancies in the Japanese patients with inflammatory bowel disease. J Gastroenterol. 2014;49(9):1299-306.

5 Van der Woude CJ, Ardizzone S, Bengtson MB, Fiorino G, Fraser G, Katsanos K, et al. The second European evidenced-based consensus on reproduction and pregnancy in inflammatory bowel disease. J Crohns Colitis. 2015;9(2):107-24.

6 Sturm A, Maaser C, Mendall M, Karagiannis D, Karatzas P, Ipenburg N, et al. European Crohn's and colitis organisation topical review on IBD in the elderly. J Crohn Colitis. 2017;11(3):263-73.
7 Komoto S, Higashiyama M, Watanabe C, Suzuki Y, Watanabe M, Hibi T, et al. Clinical differences between elderly-onset ulcerative colitis and non-elderly-onset ulcerative colitis: a nationwide survey data in Japan. J Gastroenterol Hepatol. 2018;33(11):1839-43.

8 Higashiyama M, Sugita A, Koganei K, Wanatabe K, Yokoyama Y, Uchino M, et al. Management of elderly ulcerative colitis in Japan. J Gastroenterol. 2019;54(7):571-86.

9 Ito A, Omori T, Hanafusa N, Tsuchiya K, Nakamura S, Tokushige K. Efficacy and safety of granulocyte adsorption apheresis in elderly patients with ulcerative colitis. J Clin Apher. 2018;33(4):514-20.

10 Komoto S, Matsuoka K, Kobayashi T, Yokoyama Y, Suzuki Y, Hibi T, et al. Safety and efficacy of leukocytapheresis in elderly patients with ulcerative colitis: the impact in steroid-free elderly patients. J Gastroenterol Hepatol. 2018;33(8):1485-91.

11 Ungaro RC, Aggarwal S, Topaloglu O, Lee WJ, Clark R, Colombel JF. Systematic review and meta-analysis: efficacy and safety of early biologic treatment in adult and paediatric patients with Crohn's disease. Aliment Pharmacol Ther. 2020;51(9):831-42.

12 Osterman MT, Sandborn WJ, Colombel JF, Robinson AM, Lau W, Huang B, et al. Increased risk of malignancy with adalimumab combination therapy, compared with monotherapy, for Crohn's disease. Gastroenterology. 2014;146(4):941-9.
13 Axelrad J, Bernheim O, Colombel JF, Malerba S, Ananthakrishnan A, Yajnik V, et al. Risk of new or recurrent cancer in patients with inflammatory bowel disease and previous cancer exposed to immunosuppressive and antitumor necrosis factor agents. Clin Gastroenterol Hepatol. 2016;14(1):58-64.

14 Matsumoto T, Yanai S, Toya Y, Ueno M, Nakamura S. Internet-orientated assessment of QOL and actual treatment status in Japanese patients with inflammatory bowel disease: the 3I survey. J Crohns Colitis. 2015;9(6):477-82.

15 Ueno F, Nakayama Y, Hagiwara E, Kurimoto S, Hibi T. Impact of inflammatory bowel disease on Japanese patients' quality of life: results of a patient questionnaire survey. J Gastroenterol. 2017;52(5):555-67.

16 Neuendorf R, Harding A, Stello N, Hanes D, Wahbeh H. Depression and anxiety in patients with inflammatory bowel disease: a systematic review. J Psychosom Res. 2016;87: $70-80$.

17 Takahashi M, Aoyama N, Nunotani M. N03 Disease burden of patients with inflammatory bowel disease from the viewpoint of QOL and depression. J Crohn Colitis. 2020;14(Suppl 1): S658-9.

18 Le Berre C, Flamant M, Bouguen G, Siproudhis L, Dewitte M, Dib N, et al. Validation of the IBD-Disk instrument for assessing disability in inflammatory bowel diseases in a French cohort: the VALIDate study. J Crohn Colitis. 2020:jjaa100. 